\title{
2378. Adaptive backstepping sliding mode control for heavy-weight airdrop operations
}

\author{
Ri Liu, Xiuxia Sun, Wenhan Dong, Xuning Guan \\ 1,2,3 Air Force Engineering University, Xi'an, 710038, China \\ ${ }^{4}$ The First Aeronautical College of Air Force, Xinyang, 464000, China \\ ${ }^{3}$ Corresponding author \\ E-mail: 1r_taiyang@yeah.net, ${ }^{2}$ gcxysxx@126.com,3idongwenhan@sina.com, ${ }^{4} a f$ gxn@126.com
}

Received 15 October 2016; received in revised form 5 December 2016; accepted 27 December 2016 DOI https://doi.org/10.21595/jve.2016.17838

Check for updates

\begin{abstract}
This paper proposes an adaptive backstepping sliding mode flight control method that is compatible with heavyweight cargo airdrop. The goal is to maintain the plane states during cargo extraction process, in the presence of uncertainties of both constant and time-varying types, as well as matched and unmatched types. A backstepping sliding mode flight control law with parameter adaptation is presented based on the plane-cargo dynamics in strict-feedback form. The control approach consists in having an adaptation law that approximates the disturbance and uncertain aerodynamic function, which is separated from the complex nonlinearities. Also, the adaptation algorithm with projection can bound the estimated function. This ensures the robustness of the controller against time-varying disturbance and uncertainty. The convergence performance and robustness property of the control law are proved by the Lyapunov theory. The control effect is evaluated on a transport plane performing a maximum load airdrop task in a number of simulation scenarios.
\end{abstract}

Keywords: flight control, flight dynamics, time-varying uncertainty, adaptive control, backstepping control, sliding mode control.

\section{Introduction}

Heavyweight cargo airdrop is a main function of a transport plane, and it has been widely used in modern military tasks and humanitarian aid [1-3]. The successful release and landing of heavy payloads greatly depends on the operations of the flight control system of the plane, which is imperative to reject sudden and large disturbances caused by the heavy cargo and also to accommodate various system uncertainties $[4,5]$.

Over recent years, some achievements have been reported in developing advanced flight control laws that are compatible with the heavyweight cargo airdrop. Based on the linear system at a given trimming position, [6] investigated the $L_{1}$ adaptive control approach subject to system uncertainties, [7] and [8] made a research on the use of active disturbance rejection control technique, and [9] proposed a robust control approach to achieve specified handing qualities. A key problem is that thus linear design controllers may cause unsatisfactory performance in the event that the cargo becomes increasingly heavy. In such an event, the plane dynamics would deviate far from the operating point in cargo extraction, thus leading to a highly nonlinear system. It is interesting to linearize the model at a train of operating points throughout the airdrop process [9], but this is really a tedious work and cannot fundamentally solve the aforementioned problem. Many nonlinear control methods have been proposed to overcome the shortcomings of the linear design controllers. The theoretically established feedback linearization method is the one that is most widely applied [10].

A nonlinear system can be transformed into a constant linear system rather than linear approximations via nonlinear feedback and exact state transformations. However, precise knowledge of the plant model must be known for achieving perfect feedback linearization. It is generally not the case for the airdrop flight controller design, as the complex nonlinear aerodynamic characteristics are very difficult to ascertain and model precisely [5, 6, 11]. Moreover, aerodynamic data obtained from wind tunnel tests always contain a certain degree of 
uncertainty. Sliding mode control (SMC) is an efficient method to deal with model deficiencies. Based on the feedback linearization model of the airdrop process, a preliminary research on the use of linear SMC techniques to stabilize the plane speed and pitch attitude is introduced in [12], and an iterative SMC strategy that integrates the merits of the global sliding mode and integral sliding mode is proposed in [11]. In spite of their interesting ideas, these methods require the upper bounds of the uncertainties to specify the control gains to satisfy the requirement of stability and robustness. However, the complex uncertainties are always unavailable. In this case, the control gains are usually required to be selected large enough to operate correctly under a variety of conditions, which is generally a very conservative strategy [13], and might result in severe chattering phenomena that even damage systems and actuators [14].

Additionally, the aforementioned approaches cannot handle unmatched uncertainties. This also limits their implementations in the design of airdrop flight controllers from a purely practical perspective. Backstepping control is a recursive method for stabilizing systems with both matched and unmatched uncertainties. Combining with the sliding mode method, authors of [15] designed a backstepping SMC law, which solves the unmatched uncertain control problem for cargo airdrop, but their approach still has the conservative problem as mentioned above. As the motivation is the non-requirement of the bounds on the complex nonlinear uncertainties, the idea of employing adaptation algorithms to approximate the uncertainties have attracted much attention. Papers [16-18] developed a class of online learning algorithm which is compatible with uncertainties estimation of complex systems. Authors of $[19,20]$ proposed a novel estimation method based on swarm intelligence optimization algorithms, and this method also provide a feasible solution to deal with uncertainties of complex plants. Papers [21, 22] introduced neural networks to parameterize the complex uncertainties in designing adaptive backstepping controller, so that the update laws can adapt the network weights. Paper [23] separated the uncertain parameters from the complex nonlinearities and directly estimated the uncertain parameters using adaptation algorithms. The design procedure, as well as the performance analysis of such an approach, is relatively easy when compared with that using the neural networks method.

The main motivation for this work is to present a flight controller design that can accommodate large changes in plane dynamics and reject uncertainties of both constant and time-varying types, as well as matched and unmatched types. The contributions include 1) a flight controller design that inherits the merits of the SMC and backstepping methods, thus solving the unmatched control problem of cargo airdrop; 2) the employment of adaptation techniques to approximate the disturbance and uncertain aerodynamic function, which is separated from the complex nonlinearities; 3 ) the formation of adaptation laws using the projection operator to bound the estimated function $[24,25]$, which theoretically ensures the robustness of the controller against time-varying uncertainties; and 4) a proof of the convergence performance and robustness property of the control approach based on the Lyapunov theory.

\section{Plane-cargo dynamics}

The airdrop process starts as the plane adjusts to a steady flight at the target area. The cargo moves along the rail and is pulled out of the cabin with the help of the extraction system, as shown in Fig. 1. We assumed that the cargo is a particle and the rail coincides with the longitudinal axis of the plane. Thus, the cargo only affects the lateral dynamics of the plane. The disturbances that the cargo imposes on the plane are the contact force $F_{c z}$, friction force $F_{c x}$, and additional moment $M_{c}$. The aerodynamic forces imposing on the plane include $L$ and $D$, which are the lift and drag forces. The thrust and pitch aerodynamic moment are denoted by $T$ and $M_{y}$, respectively. The pitch flight is characterized by the airspeed $V$, climb angle $\gamma$, attack angle (AOA) $\alpha$, and pitch angle $\theta$, respectively.

The plane dynamics during airdrop are as follows [11]: 
$\dot{V}=\frac{\left(T \cos \alpha-D-m_{b} g \sin \gamma-F_{c x}\right)}{m_{b}}$,

$\dot{\gamma}=\left(T \sin \alpha+L-m_{b} g \cos \gamma-F_{c z} \frac{)}{m_{b} V^{\prime}}\right.$

$\dot{q}=\frac{\left(M_{y}+M_{c}\right)}{I_{y}}$,

$\dot{\theta}=q$,

where $q$ stands for the pitch rate; $m_{b}$ stands for the mass of the plane; and $I_{y}$ stands for the pitch moment of inertia.

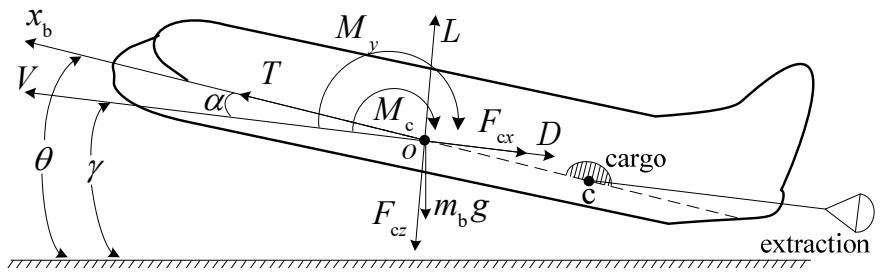

Fig. 1. Forces analysis of the plane during airdrop

The lift and drag forces are found by:

$D=\bar{q} S\left[C_{D 0}+C_{D \alpha}\left(\alpha-\alpha_{0}\right)+C_{D \delta_{e}} \delta_{e}\right]$,

$L=\bar{q} S\left[C_{L 0}+C_{L \alpha}\left(\alpha-\alpha_{0}\right)+C_{L \delta_{e}} \delta_{e}\right]$,

where $\bar{q}$ stands for the dynamic pressure; $S$ stands for the wing area; $\delta_{e}$ stands for the elevator deflection; $C_{D *}$ and $C_{L *}$ represent the drag and lift coefficients, respectively.

$M_{y}$ is given by:

$M_{y}=\bar{q} S c_{A}\left[C_{m 0}+C_{m \alpha}\left(\alpha-\alpha_{0}\right)+C_{m q} \frac{q c_{A}}{2 V}+C_{m \delta_{e}} \delta_{e}\right]$,

where $C_{m *}$ represent the pitch aerodynamic moment coefficients and $c_{A}$ stands for the mean aerodynamic chord.

The thrust force is given by:

$T=T_{m} \delta_{p}$

where $\delta_{p}$ is the throttle opening ranging from 0 to $100 \%$ and $T_{m}$ is the maximal thrust.

The expressions of $F_{c x}, F_{c z}$, and $M_{c}$ derived from the cargo dynamics. As shown in Fig. 2, the cargo dynamics can be obtained as:

$m_{c} a_{w x}=F_{c x}-F_{p}-m_{c} g \sin \gamma$,

$m_{c} a_{w z}=-F_{c z}+m_{c} g \cos \gamma$,

$F_{c x}=\mu F_{c Z}$,

where $m_{c}$ stands for the mass of the cargo; $a_{w x}$ and $a_{w z}$ are the $x$-acceleration and $z$-acceleration of the cargo in the wind-axes frame, respectively; $\mu$ stands for the coefficient of cargo to friction; and $F_{p}$ is the extraction force found by $F_{p}=m_{c} g \lambda$ with $\lambda$ denoting the extraction ratio.

Using the theorem of composition of particles' acceleration, it follows that the acceleration of the cargo is the sum of its implicated acceleration, Coriolis acceleration, and relative acceleration [11]: 
$a_{w x}=\dot{V}+\dot{q} r_{c} \sin \alpha+q^{2} r_{c} \cos \alpha+2 q \dot{r}_{c} \sin \alpha-\ddot{r}_{c} \cos \alpha$,

$a_{w z}=\dot{q} r_{c} \cos \alpha-V \dot{\gamma}-q^{2} r_{c} \sin \alpha+2 q \dot{r}_{c} \cos \alpha+\ddot{r}_{c} \sin \alpha$,

where $r_{c}$ is the distance of point $c$ to point $o$.

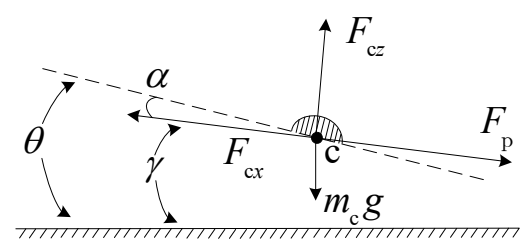

Fig. 2. Forces analysis of the cargo

Substituting Eqs. (12) and (13) into Eqs. (9) and (10) yields:

$$
\begin{aligned}
F_{c x} & =F_{p}+m_{c} r_{c} \sin \alpha \dot{q}+m_{c} \dot{V}-\left(m_{c} g \cos \theta-2 m_{c} q \dot{r}_{c}\right) \sin \alpha \\
& +\left(m_{c} g \sin \theta+m_{c} q^{2} r_{c}-m_{c} \ddot{r}_{c}\right) \cos \alpha, \\
F_{c z} & =\left(m_{c} g \cos \theta-2 m_{c} q \dot{r}_{c}\right) \cos \alpha+m_{c} V \dot{\gamma}-m_{c} r_{c} \cos \alpha \dot{q} \\
& +\left(m_{c} g \sin \theta+m_{c} q^{2} r_{c}-m_{c} \ddot{r}_{c}\right) \sin \alpha,
\end{aligned}
$$

with:

$$
\begin{aligned}
\ddot{r}_{c}= & \dot{V} \cos \alpha+V \sin \alpha \dot{\gamma}+g \sin \theta-\mu g \cos \theta+\frac{\mu F_{p} \sin \alpha}{m_{c}}+r_{c} q^{2}+\frac{F_{p} \cos \alpha}{m_{c}} \\
& +\mu\left(\dot{V} \sin \alpha-V \dot{\gamma} \cos \alpha+\dot{q} r_{c}+2 q \dot{r}_{c}\right) .
\end{aligned}
$$

From Fig. 1, the disturbance moment can be obtained as:

$$
\begin{aligned}
M_{c} & =r_{c} \cdot\left(F_{c z} \cos \alpha-F_{c x} \sin \alpha\right) \\
& =m_{c} r_{c} g \cos \theta-F_{p} r_{c} \sin \alpha-m_{c} r_{c}\left(\dot{V} \sin \alpha-V \dot{\gamma} \cos \alpha+\dot{q} r_{c}+2 q \dot{q}_{c}\right) .
\end{aligned}
$$

Remark 1. Eqs. (1)-(3) are the acceleration equation, centripetal acceleration equation, and angular acceleration equation of the plane. Eq. (4) is the pitch angle kinematic equation. It is observed from Eqs. (1)-(3) and (14)-(17) that the plane-cargo dynamics form a strongly nonlinear system subject to the coupling of the plane and cargo states. Also, the system contains various uncertainties, such as aerodynamic data perturbation. Readers can refer to [11] for detailed discussions about the model.

Combining Eqs. (1)-(17), together with the consideration of uncertainties, we can written the plane dynamics in cargo extraction as follows:

$$
\left\{\begin{array}{l}
\dot{x}_{1}(t)=\sigma(t)+B x_{2}(t), \\
\dot{x}_{2}(t)=F(t)+G(t) u(t)+\Delta F(t),
\end{array}\right.
$$

where $x_{1}=\theta, x_{2}=[V, q]^{T}, u=\left[\delta_{e}, \delta_{p}\right]^{T}, B=[0,1], F=\left[f_{1}, f_{2}\right]^{T}$, and $G=\left[\begin{array}{ll}g_{11} & g_{12} \\ g_{21} & g_{22}\end{array}\right] ; \sigma(t)$ stands for the time-varying disturbance; and $\Delta F=\left[\Delta f_{1}, \Delta f_{2}\right]^{T}$ stands for the time-varying uncertainty. $f_{i}$ and $g_{i j}(i=1,2 ; j=1,2)$ are:

$$
\begin{gathered}
f_{1}=\left[-m_{c} r_{c} \sin \alpha f_{2}+T_{0} \cos \alpha-m_{b} g \sin \gamma-\Lambda_{1} \cos \alpha\right. \\
+\frac{\left.\Lambda_{2} \sin \alpha-\bar{q} S\left(C_{D 0}+C_{D \alpha}\left(\alpha-\alpha_{0}\right)\right)-F_{p}\right]}{\left(m_{b}+m_{c}\right)}
\end{gathered}
$$




$$
\begin{aligned}
f_{2}= & \bar{q} S c_{A}\left(C_{m 0}+C_{m \alpha}\left(\alpha-\alpha_{0}\right)+C_{m q} \frac{q c_{A}}{2 V}\right) / \Lambda_{3}+\frac{r_{c} \Lambda_{2}}{\Lambda_{3}} \\
& -\frac{r_{c} F_{p} \sin \alpha}{\Lambda_{3}}+m_{c} r_{c}\left[-\Lambda_{2}+F_{p} \sin \alpha+m_{b} g \sin \gamma \sin \alpha-m_{b} g \cos \gamma \cos \alpha\right. \\
& \left.+\bar{q} S \sin \alpha\left(C_{D 0}+C_{D \alpha}\left(\alpha-\alpha_{0}\right)\right)+\bar{q} S \cos \alpha\left(C_{L 0}+C_{L \alpha}\left(\alpha-\alpha_{0}\right)\right)\right] /\left[\left(m_{b}+m_{c}\right) \Lambda_{3}\right], \\
g_{11} & =-\frac{\left(m_{c} r_{c} \sin \alpha g_{21}+\bar{q} S C_{D \delta_{e}}\right)}{\left(m_{b}+m_{c}\right)}, \\
g_{12} & =\frac{T_{m} \cos \alpha}{\left(m_{b}+m_{c}\right)}, \\
g_{21} & =\frac{m_{c} r_{c} \bar{q} S\left(C_{D \delta_{e}} \sin \alpha+C_{L \delta_{e}} \cos \alpha\right)}{\left[\left(m_{b}+m_{c}\right) \Lambda_{3}\right]}+\frac{\bar{q} S c_{A} C_{m \delta_{e}}}{\Lambda_{3}}, \\
g_{22} & =0,
\end{aligned}
$$

with $\Lambda_{i}(i=1,2,3)$ being defined as:

$$
\begin{aligned}
& \Lambda_{1}=m_{c} g \sin \theta+m_{c} q^{2} r_{c}-m_{c} \ddot{r}_{c}, \\
& \Lambda_{2}=m_{c} g \cos \theta-2 m_{c} q \dot{r}_{c}, \\
& \Lambda_{3}=I_{y}+m_{c} r_{c}^{2}-\frac{m_{c}^{2} r_{c}^{2}}{\left(m_{b}+m_{c}\right)} .
\end{aligned}
$$

The uncertainty functions $\Delta f_{i}(i=1,2)$ introduced by the aerodynamic data perturbation are obtained as:

$$
\begin{aligned}
& \Delta f_{1}=\frac{\left[-m_{c} r_{c} \sin \alpha \Delta f_{2}-\bar{q} S\left(\Delta C_{D 0}+\Delta C_{D \alpha}\left(\alpha-\alpha_{0}\right)\right)\right]}{\left(m_{b}+m_{c}\right)}, \\
& \Delta f_{2}=\bar{q} S c_{A}\left(\Delta C_{m 0}+\Delta C_{m \alpha}\left(\alpha-\alpha_{0}\right)+\Delta C_{m q} \frac{q c_{A}}{2 V}\right) / \Lambda_{3} \\
& +m_{c} r_{c} \bar{q} S\left[\sin \alpha\left(\Delta C_{D 0}+\Delta C_{D \alpha}\left(\alpha-\alpha_{0}\right)\right)\right. \\
& \left.+\cos \alpha\left(\Delta C_{L 0}+\Delta C_{L \alpha}\left(\alpha-\alpha_{0}\right)\right)\right] /\left[\left(m_{b}+m_{c}\right) \Lambda_{3}\right] \text {, }
\end{aligned}
$$

where $\Delta C_{D *}, \Delta C_{L^{*}}$, and $\Delta C_{m *}$ denote the uncertainties of the drag, lift, and pitch aerodynamic moment coefficients, respectively. By introducing the following notations:

$$
\begin{aligned}
& E_{11}=-\frac{\bar{q} S m_{c}^{2} r_{c}^{2} \sin \alpha \cos \alpha}{\left[\left(m_{b}+m_{c}\right)^{2} \Lambda_{3}\right]} \\
& E_{12}=-\frac{\bar{q} S}{\left(m_{b}+m_{c}\right)}-\frac{\bar{q} S m_{c}^{2} r_{c}^{2} \sin ^{2} \alpha}{\left[\left(m_{b}+m_{c}\right)^{2} \Lambda_{3}\right]} \\
& E_{13}=-\frac{\bar{q} S c_{A} m_{c} r_{c} \sin \alpha}{\left[\left(m_{b}+m_{c}\right) \Lambda_{3}\right]} \\
& E_{21}=\frac{\bar{q} S m_{c} r_{c} \cos \alpha}{\left[\left(m_{b}+m_{c}\right) \Lambda_{3}\right]} \\
& E_{22}=\frac{\bar{q} S m_{c} r_{c} \sin \alpha}{\left[\left(m_{b}+m_{c}\right) \Lambda_{3}\right]} \\
& E_{23}=\frac{\bar{q} S c_{A}}{\Lambda_{3}} \text {, } \\
& E(t)=\left[\begin{array}{lll}
E_{11} & E_{12} & E_{13} \\
E_{21} & E_{22} & E_{23}
\end{array}\right] \cdot\left[\begin{array}{ccccccc}
1 & \alpha-\alpha_{0} & 0 & 0 & 0 & 0 & 0 \\
0 & 0 & 1 & \alpha-\alpha_{0} & 0 & 0 & 0 \\
0 & 0 & 0 & 0 & 1 & \alpha-\alpha_{0} & q c_{A} / 2 V
\end{array}\right], \\
& P(t)=\left[\Delta C_{L 0}, \Delta C_{L \alpha}, \Delta C_{D 0}, \Delta C_{D \alpha}, \Delta C_{m 0}, \Delta C_{m \alpha}, \Delta C_{m q}\right]^{T} \text {, }
\end{aligned}
$$


the uncertainty $\Delta F(t)$ can be written as:

$\Delta F(t)=E(t) P(t)$.

Assumption 1. (Uniform boundedness of the uncertainties): $|\sigma(t)| \leq \phi$ and $P(t) \in \Theta$, where $\phi>0$ is a known bound of $\sigma(t)$ and $\Theta$ is a known compact set.

Assumption 2. (Uniform boundedness of the rate of variation of the uncertainties): $\sigma(t)$ and $P(t)$ are continuously differentiable with uniformly bounded derivatives, i.e., $|\dot{\sigma}(t)| \leq d_{\sigma}<\infty$ and $\|\dot{P}(t)\| \leq d_{P}<\infty$ with $\|(\cdot)\|$ being the 2-norm of the vector.

Assumption 3. [11] The control input matrix $G(t)$ is nonsingular within the scope of $-\pi / 2<\alpha<\pi / 2$.

\section{Control law and convergence performance}

As shown in Fig. 3, the overall control system contains three feedback loops. In the outer loop, an altitude controller is designed by the use of the proportion-differentiation (PD) method. This loop generates a command $\theta_{d}$ for the pitch angle controller in the second layer. The controlled variables of the inner loop are $[q, V]$, and the pitch rate command $q_{d}$ is generated by pitch angle controller. The altitude and airspeed commands are characterized by $H_{d}$ and $V_{d}$, which are defined as those of the trimming position. The design of the pitch angle, pitch rate and speed controllers is the main work.

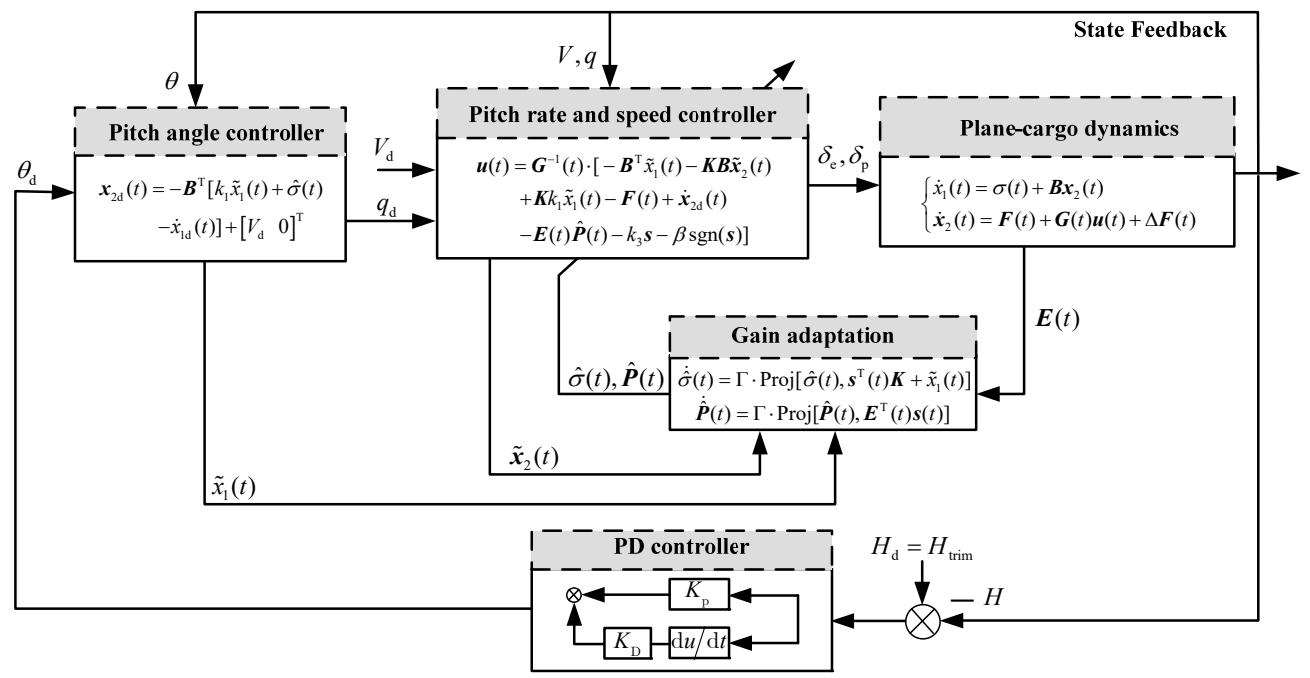

Fig. 3. Autopilot control architecture with three layers of feedback

First, consider the first subsystem in Eq. (18), which is rewritten here for convenience:

$\dot{x}_{1}(t)=\sigma(t)+B x_{2}(t)$.

We define:

$\tilde{x}_{1}(t)=x_{1}(t)-x_{1 d}(t)$

where $x_{1 d}(t)=\theta_{d}$ and $\tilde{x}_{1}(t)$ is the tracking error of $x_{1}(t)$. We can design the control law of Eq. (39) as:

$x_{2 d}(t)=-B^{T}\left[k_{1} \tilde{x}_{1}(t)+\hat{\sigma}(t)-\dot{x}_{1 d}(t)\right]+\left[V_{d} 0\right]^{T}$, 
where $k_{1}>0$, and $\hat{\sigma}(t)$ stands for the approximation to $\sigma(t)$.

Then, consider the second subsystem in Eq. (18), which is rewritten here for convenience:

$\dot{x}_{2}(t)=F(t)+G(t) u(t)+E(t) P(t)$.

We define the tracking error of $x_{2}(t)$ as:

$\tilde{x}_{2}(t)=x_{2}(t)-x_{2 d}(t)$

We then define sliding manifolds:

$s(t)=K \tilde{x}_{1}(t)+\tilde{x}_{2}(t)$

to shape the tracking errors. Here $K=\left[0, k_{2}\right]^{T}$ with $k_{2}>0$. We design the control law as:

$$
\begin{aligned}
& u(t)=G^{-1}(t) \cdot\left[-B^{T} \tilde{x}_{1}(t)-K B \tilde{x}_{2}(t)+K k_{1} \tilde{x}_{1}(t)-F(t)\right. \\
& \left.\quad+\dot{x}_{2 d}(t)-\mathrm{E}(t) \hat{P}(t)-k_{3} s-\beta \operatorname{sgn}(\mathrm{s})\right]
\end{aligned}
$$

where $k_{3}, \beta>0$, and $\hat{P}(t)$ stands for the approximation to $P(t)$.

$\hat{\sigma}(t)$ and $\hat{P}(t)$ are governed by the following adaptation laws:

$\dot{\hat{\sigma}}(t)=\Gamma \cdot \operatorname{Proj}\left[\hat{\sigma}(t), s^{T}(t) K+\tilde{x}_{1}(t)\right]$,

$\dot{\hat{P}}(t)=\Gamma \cdot \operatorname{Proj}\left[\hat{P}(t), E^{T}(t) s(t)\right]$,

where $\Gamma>0$ is the adaptation gain and $\operatorname{Proj}(\cdot, \cdot)$ is the projection operator (see Appendix for details) which ensures that $|\hat{\sigma}| \leq \phi, \hat{P}(t) \in \Theta$.

Theorem 1. Given the system defined in Eq. (18) controlled by Eq. (45) with the sliding manifold in Eq. (44) and the adaptation laws in Eqs. (46) and (47), the states tracking errors $\tilde{x}_{1}(t)$ and $\tilde{x}_{2}(t)$ are bounded as:

$\tilde{x}_{1}(t), \tilde{x}_{2}(t) \in\left\{\tilde{x}_{1}{ }^{2}+\|s\|^{2}<\frac{2 l}{\Gamma}\right\}, \quad \forall t>0$,

where:

$l=\frac{1}{k_{\min }}\left(d_{\sigma} \phi+\max _{P \in \Theta}\|P\| d_{P}\right)+2\left(\phi^{2}+\max _{P \in \Theta}\|P\|^{2}\right)$

with $k_{\min }=\min \left\{k_{3}, k_{1}+k_{2}\right\}$.

Proof. To proceed with the proof, we define the approximator parameter errors as:

$\tilde{\sigma}(t)=\hat{\sigma}(t)-\sigma(t)$

$\tilde{P}(t)=\hat{P}(t)-P(t)$.

The tracking error dynamics of $x_{1}(t)$ and $x_{2}(t)$ can be obtained as:

$$
\begin{aligned}
& \dot{\tilde{x}}_{1}(t)=\dot{x}_{1}(t)-\dot{x}_{1 d}(t)=\sigma(t)+B x_{2}(t)-\dot{x}_{1 d}(t) \\
& \quad=\sigma(t)-k_{1}\left(x_{1}(t)-x_{1 d}(t)\right)-\hat{\sigma}(t)+B \tilde{x}_{2}(t)=-k_{1} \tilde{x}_{1}(t)-\tilde{\sigma}(t)+B \tilde{x}_{2}(t), \\
& \dot{\tilde{x}}_{2}(t)=\dot{x}_{2}(t)-\dot{x}_{2 d}(t)=F(t)+G(t) u(t)+E(t) P(t)-\dot{x}_{2 d}(t) \\
& \quad=-E(t) \tilde{P}(t)-B^{T} \tilde{x}_{1}(t)-K B \tilde{x}_{2}(t)+K k_{1} \tilde{x}_{1}(t)-k_{3} s-\beta \operatorname{sgn}(\mathrm{s}) .
\end{aligned}
$$

Consider the Lyapunov candidate function: 
$v(t)=\frac{1}{2} s^{T} s+\frac{1}{2} \tilde{x}_{1}{ }^{2}+\frac{1}{2 \Gamma}\left(\tilde{\sigma}^{2}+\tilde{P}^{T} \tilde{P}\right)$

We first prove $v(t) \leq \frac{l}{\Gamma}$. Since the plane should maintain a straight and level flight condition before the cargo is unlocked $[4-6,8,9]$, we can write $\tilde{x}_{i}(0)=0$ for $i=1,2$. Also, we have:

$v(0) \leq \frac{1}{2 \Gamma}\left(4 \phi^{2}+4 \max _{P \in \Theta}\|P\|^{2}\right) \leq \frac{l}{\Gamma}$

Taking the derivative of $v(t)$ along the solutions of Eqs. (52) and (53) yields:

$$
\begin{aligned}
\dot{v}(t) & =s^{T} \dot{s}+\tilde{x}_{1} \dot{\tilde{x}}_{1}+\frac{1}{\Gamma}\left[\tilde{\sigma}(t) \dot{\hat{\sigma}}(t)+\tilde{P}^{T}(t) \dot{\hat{P}}(t)\right]-\frac{1}{\Gamma}\left[\tilde{\sigma}(t) \dot{\sigma}(t)+\tilde{P}^{T}(t) \dot{P}(t)\right] \\
& =s^{T}\left[K\left(-k_{1} \tilde{x}_{1}(t)-\tilde{\sigma}(t)+B \tilde{x}_{2}(t)\right)-E(t) \tilde{P}(t)-B^{T} \tilde{x}_{1}(t)-K B \tilde{x}_{2}(t)\right. \\
& \left.+K k_{1} \tilde{x}_{1}(t)-k_{3} s-\beta \operatorname{sgn}(s)\right]+\tilde{x}_{1}\left[-k_{1} \tilde{x}_{1}(t)-\tilde{\sigma}(t)+B \tilde{x}_{2}(t)\right] \\
& +\frac{1}{\Gamma}\left[\tilde{\sigma}(t) \dot{\hat{\sigma}}(t)+\tilde{P}^{T}(t) \dot{\hat{P}}(t)\right]-\frac{1}{\Gamma}\left[\tilde{\sigma}(t) \dot{\sigma}(t)+\tilde{P}^{T}(t) \dot{P}(t)\right] \\
& =-s^{T} K \tilde{\sigma}(t)-s^{T} E(t) \tilde{P}(t)-s^{T} B^{T} \tilde{x}_{1}(t)-k_{3} s^{T} s-\beta s^{T} \operatorname{sgn}(s)-k_{1} \tilde{x}_{1}^{2}(t) \\
& -\tilde{x}_{1} \tilde{\sigma}(t)+\tilde{x}_{1} B \tilde{x}_{2}(t)+\frac{1}{\Gamma}\left[\tilde{\sigma}(t) \dot{\hat{\sigma}}(t)+\tilde{P}^{T}(t) \dot{\hat{P}}(t)\right]-\frac{1}{\Gamma}\left[\tilde{\sigma}(t) \dot{\sigma}(t)+\tilde{P}^{T}(t) \dot{P}(t)\right] \\
& =-\left(k_{1}+k_{2}\right) \tilde{x}_{1}^{2}(t)-k_{3} s^{T} s-\beta s^{T} \operatorname{sgn}(s) \\
& +\tilde{\sigma}(t)\left[\frac{1}{\Gamma} \dot{\hat{\sigma}}(t)-\left(s^{T} K+\tilde{x}_{1}(t)\right)\right]+\tilde{P}^{T}(t)\left[\frac{1}{\Gamma} \dot{\hat{P}}(t)-E^{T}(t) s\right] \\
& -\frac{1}{\Gamma}\left[\tilde{\sigma}(t) \dot{\sigma}(t)+\tilde{P}^{T}(t) \dot{P}(t)\right] .
\end{aligned}
$$

Substituting Eqs. (46) and (47) into Eq. (56) leads to:

$$
\begin{aligned}
& \dot{v}(t)=-\left(k_{1}+k_{2}\right) \tilde{x}_{1}^{2}(t)-k_{3} s^{T} s-\beta s^{T} \operatorname{sgn}(s) \\
& \quad+\tilde{\sigma}(t)\left[\operatorname{Proj}\left(\hat{\sigma}(t), s^{T}(t) K+\tilde{x}_{1}(t)\right)-\left(s^{T} K+\tilde{x}_{1}(t)\right)\right] \\
& \quad+\tilde{P}^{T}(t)\left[\operatorname{Proj}\left(\hat{P}(t), E^{T}(t) s(t)\right)-E^{T}(t) s\right]-\frac{1}{\Gamma}\left[\tilde{\sigma}(t) \dot{\sigma}(t)+\tilde{P}^{T}(t) \dot{P}(t)\right] .
\end{aligned}
$$

Property A2 (see Appendix for details) implies that:

$$
\begin{aligned}
& \tilde{\sigma}(t)\left[\operatorname{Proj}\left(\hat{\sigma}(t), s^{T}(t) K+\tilde{x}_{1}(t)\right)-\left(s^{T} K+\tilde{x}_{1}(t)\right)\right] \leq 0, \\
& \tilde{P}^{T}(t)\left[\operatorname{Proj}\left(\hat{P}(t), E^{T}(t) s(t)\right)-E^{T}(t) s\right] \leq 0,
\end{aligned}
$$

which yields:

$$
\begin{gathered}
\dot{v}(t) \leq-\left(k_{1}+k_{2}\right) \tilde{x}_{1}^{2}(t)-k_{3} s^{T} s-\frac{1}{\Gamma}\left[\tilde{\sigma}(t) \dot{\sigma}(t)+\tilde{P}^{T}(t) \dot{P}(t)\right] \\
\leq-\left(k_{1}+k_{2}\right) \tilde{x}_{1}^{2}(t)-k_{3} s^{T} s+\frac{1}{\Gamma}\left|\tilde{\sigma}(t) \dot{\sigma}(t)+\tilde{P}^{T}(t) \dot{P}(t)\right| .
\end{gathered}
$$

It follows from $|\hat{\sigma}(t)| \leq \phi$ and $\hat{P}(t) \in \Theta$ that:

$$
\frac{1}{\Gamma}\left|\tilde{\sigma}(t) \dot{\sigma}(t)+\tilde{P}^{T}(t) \dot{P}(t)\right| \leq \frac{2}{\Gamma}\left(d_{\sigma} \phi+\max _{P \in \Theta}\|P\| d_{P}\right) .
$$

Suppose there exists a $t_{1}>0$ so that $v\left(t_{1}\right)>\frac{l}{\Gamma}$. Because: 
$\frac{1}{2 \Gamma}\left[\tilde{\sigma}^{2}(t)+\tilde{P}^{T}(t) \tilde{P}(t)\right] \leq \frac{1}{2 \Gamma}\left(4 \phi^{2}+4 \max _{P \in \Theta}\|P\|^{2}\right)$,

it then follows from Eq. (49) that:

$\frac{1}{2} s^{T} s+\frac{1}{2} \tilde{x}_{1}^{2}>\frac{1}{k_{\min } \Gamma}\left(d_{\sigma} \phi+\max _{P \in \Theta}\|P\| d_{P}\right)$,

which further leads to:

$k_{\min } s^{T} s+k_{\min } \tilde{x}_{1}^{2}>\frac{2}{\Gamma}\left(d_{\sigma} \phi+\max _{P \in \Theta}\|P\| d_{P}\right)$.

Combining Eqs. (60), (61), and (64), the $v(t)$ dynamics is such that:

$\dot{v}(t)<0$.

Since $v(0) \leq \frac{l}{\Gamma}$, it follows that $v(t) \leq \frac{l}{\Gamma}$ for every $t \geq 0$. Note that if $\frac{1}{2} \tilde{x}_{1}^{2}+\frac{1}{2}\|s\|^{2}<v(t)$, then $\tilde{x}_{1}{ }^{2}+\|s\|^{2}<\frac{2 l}{\Gamma}$. This completes the proof.

Remark 2. Such a projection-based adaptation law can bound the estimated function. This theoretically ensures the robustness of the controller against time-varying disturbance and uncertainty.

Remark 3. It follows from Eq. (48) that one can obtain the arbitrary desired tracking performance by increasing the adaptation gain $\Gamma$. However, a big gain yields large control power, which can also lead to control signal oscillations. Thus, the adaptation gain needs to be tuned using trial and error.

\section{Simulation results}

We simulate a $24,955 \mathrm{~kg}$ transport plane airdrop with an $8,000 \mathrm{~kg}$ cargo for example. The cargo is initially fixed at the center of gravity of the plane. The trimming position of the plane is as follows: the altitude $H_{0}=100 \mathrm{~m}$, airspeed $V_{0}=80 \mathrm{~m} / \mathrm{s}$, AOA $\alpha_{0}=\theta_{0}=3.8134 \mathrm{deg}$, elevator $\delta_{e}=0 \mathrm{deg}$, and throttle opening $\delta_{p}=27.1 \%$.
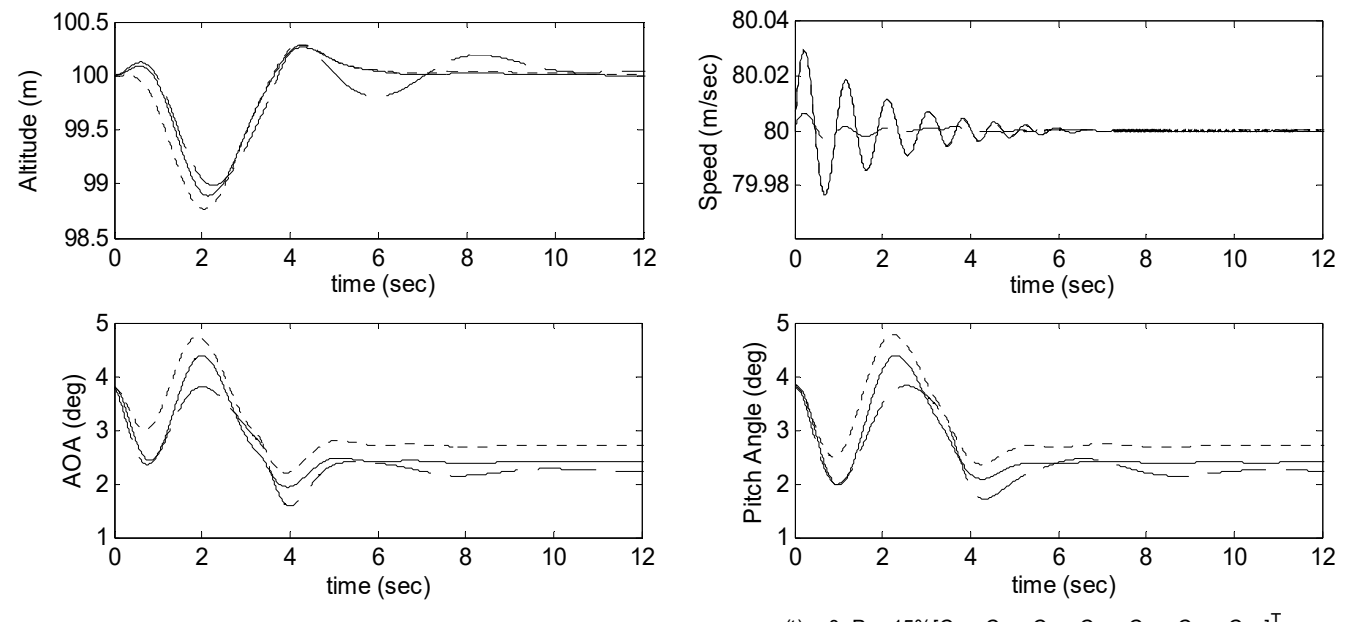

$-\cdots \sigma(\mathrm{t})=0, \mathrm{P}=0 ; \quad-\sigma(\mathrm{t})=0.01 \mathrm{rad} / \mathrm{s}, \mathrm{P}=0$; $\sigma(t)=0, P=15 \%\left[C_{L 0}, C_{L_{\alpha}}, C_{D 0}, C_{D_{\alpha}}, C_{m 0}, C_{m \alpha}, C_{m q}\right]^{\top}$

Fig. 4. The responses of the plane in cargo extraction in the presence of constant uncertainty 
The parameters of the proposed adaptive backstepping SMC are set as $k_{1}=1, k_{2}=0.5$, $k_{3}=1, \beta=0.001$, and $\Gamma=0.5$ after experimental tuning. The compact sets are conservatively set to $\phi=0.3, \Theta=\left\{b=\left(b_{i}\right)_{7 \times 1} \in R^{7 \times 1}: b_{i} \in[-2,2]\right\}$. The outer-loop altitude-hold PD control parameters are selected as $K_{p}=0.05$ and $K_{D}=0.02$. To test the requirements of task performance and flight safety, the evaluation criteria for the heavyweight airdrop can be given as [11]: (1) $|\Delta H| \leq 13 \mathrm{~m}$; (2) $|\Delta \theta| \leq 5 \mathrm{deg}$; (3) $|\Delta V| \leq 0.13 V_{0}$; and (4) $\alpha \leq 0.7 \alpha_{s}$ with $\alpha_{s}$ being the stalling AOA. The performance and robustness of the controller is first tested in the presence of constant aerodynamic uncertainty. As shown in Fig. 4, for all the three scenarios, the altitude and speed are maintained at the trimming position and fully stabilized at about $10 \mathrm{~s}$ and $6 \mathrm{~s}$, respectively. The final pitch angle becomes smaller compared to that of the trimming position due to the loss of heavy weight. Note that the observed change in value of the pitch angle is less than $5 \mathrm{deg}$. The criteria for a successful drop have all been met for the three scenarios. Fig. 5 illustrates that the throttle opening and elevator deflection remain within the limits and they do not occur severe chattering phenomenon. Overall, the pitch up movement of the plane caused by the releasing of the payload is effectively restrained through regulating the throttle and elevator appropriately.
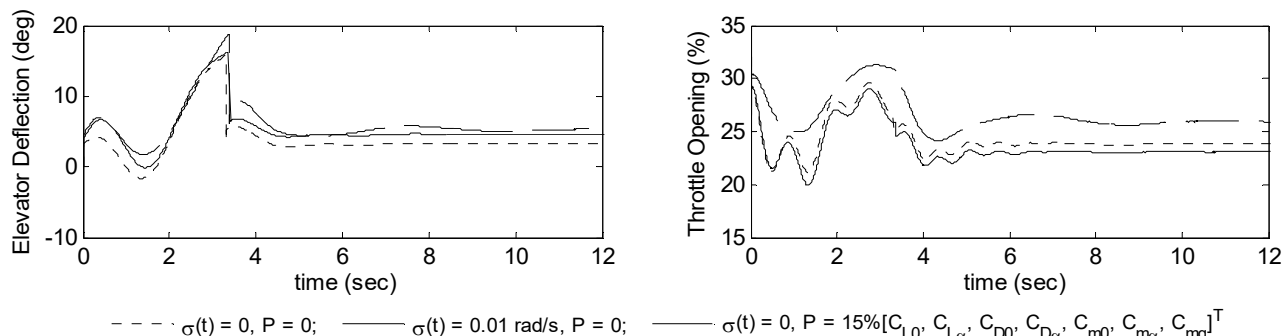

Fig. 5. Curves of the elevator and throttle in the presence of constant uncertainty
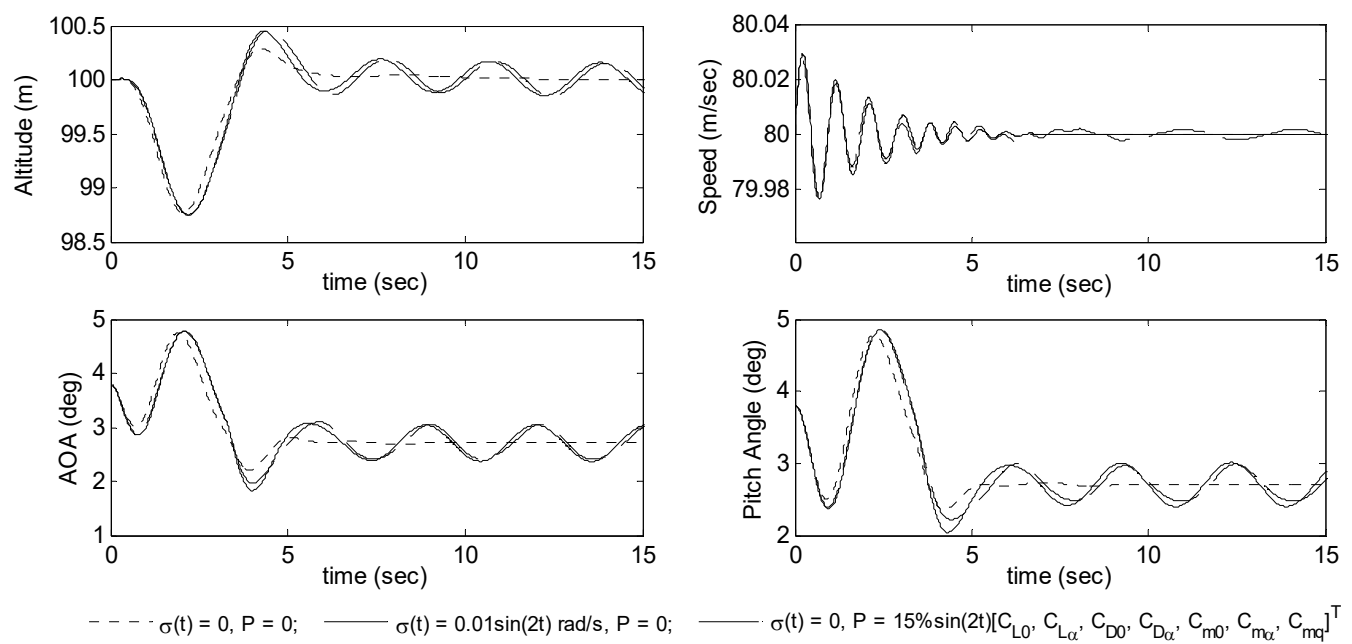

Fig. 6. The response of the plane in cargo extraction in the presence of time-varying uncertainty

Next, we test the robustness of the controller against time-varying uncertainty, without updating the control parameters. It is observed from Fig. 6 that the altitude increment is controlled within $0.3 \mathrm{~m}$, the speed is finally almost stabilized at the trimming position, and the AOA as well as the pitch angle converges to within [2.5 deg, $3 \mathrm{deg}$ ] in the presence of setting the pitch rate disturbance as $0.01 \sin (2 t) \mathrm{rad} / \mathrm{s}$. While we change the aerodynamic uncertainty by $15 \% \sin (2 t)$, the responses of the plane are similar to the previous scenario, which also satisfy the task 
performance evaluation criteria. The elevator deflection and throttle opening change periodically to reject the time-varying uncertainty, and there is no severe chattering, as presented in Fig. 7.
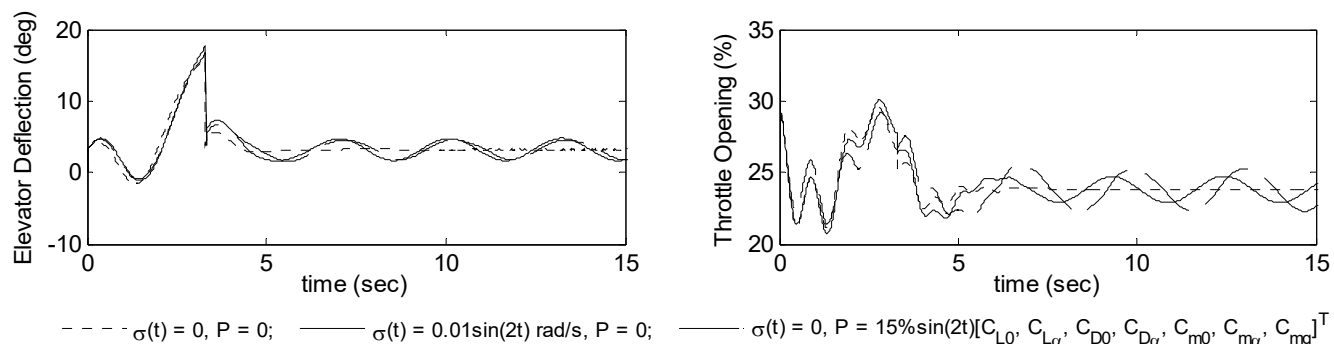

Fig. 7. Curves of the elevator and throttle in the presence of time-varying uncertainty
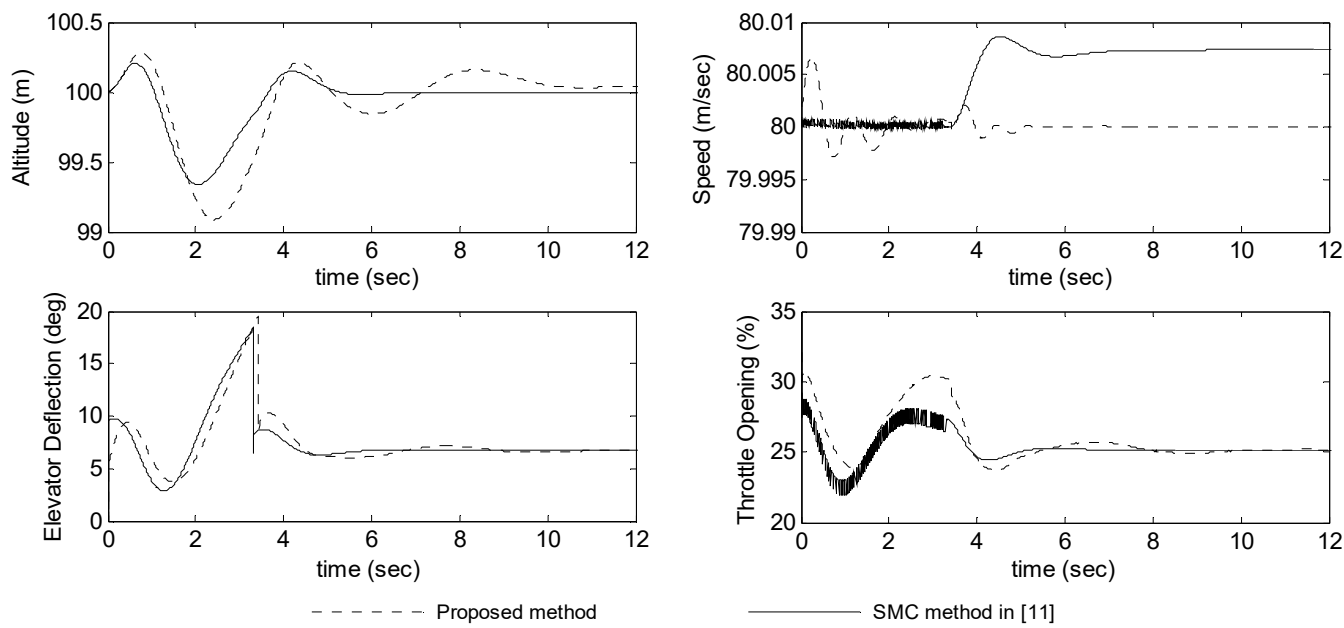

Fig. 8. Responses comparison of the dropping process with the proposed method and the SMC method [11] (in the presence of $0.01 \mathrm{rad} / \mathrm{s}$ pitch rate disturbance and $15 \%$ aerodynamic coefficients uncertainty)

We further make a comparison of control performance between the proposed control method and the SMC method in [11], in the presence of $0.01 \mathrm{rad} / \mathrm{s}$ pitch rate disturbance and $15 \%$ aerodynamic coefficients uncertainty. The sliding mode controller is recalled from [11] as following:

$$
\begin{aligned}
u & =\left[\begin{array}{ll}
b_{11} & b_{12} \\
b_{31} & b_{32}
\end{array}\right]^{-1}\left(-\left[\begin{array}{l}
f_{1} \\
f_{3}
\end{array}\right]+\left[\begin{array}{l}
\dot{V}_{d} \\
\ddot{\theta}_{d}
\end{array}\right]-\left[\begin{array}{c}
0 \\
\zeta_{2}\left(\dot{\theta}-\dot{\theta}_{d}\right)
\end{array}\right]\right. \\
& +\left[\begin{array}{c}
-\xi_{1} e^{-\xi_{1} t}\left(V(0)-V_{d}(0)\right) \\
-\xi_{2} e^{-\xi_{2} t}\left[\zeta_{2}\left(\theta(0)-\theta_{d}(0)\right)+\dot{\theta}(0)-\dot{\theta}_{d}(0)\right]
\end{array}\right] \\
& \left.-\left[\begin{array}{l}
k_{1} s_{11} e^{-\lambda_{1}\left|s_{11}\right|} \\
k_{2} s_{12} e^{-\lambda_{2}\left|s_{12}\right|}
\end{array}\right]-\left[\begin{array}{l}
\eta_{1} \operatorname{sat}\left(\left\|S_{2}\right\| / \beta\right) \cdot\left(s_{21} /\left\|S_{2}\right\|\right) \\
\eta_{2} \operatorname{sat}\left(\left\|S_{2}\right\| / \beta\right) \cdot\left(s_{22} /\left\|S_{2}\right\|\right)
\end{array}\right]\right),
\end{aligned}
$$

where $\xi_{1}, \xi_{2}, \lambda_{1}, \lambda_{2}>0$ are exponentially approaching constants; $\eta_{1}, \eta_{2}$ are robust terms which need to be chosen larger than the bounds of the system uncertainties; $k_{1}, k_{2}$ and $\zeta_{2}$ are positive constants; $s_{11}, s_{12}, s_{21}$ and $s_{22}$ are sliding manifolds with $S_{2}=\left[s_{21}, s_{22}\right]^{T} ; \beta$ is the boundary layer thickness; and $\operatorname{sat}(\cdot)$ is defined as: 
$\operatorname{sat}\left(\frac{\left\|S_{2}\right\|}{\beta}\right) \frac{S_{2}}{\left\|S_{2}\right\|}=\left\{\begin{array}{l}\operatorname{sgn}\left(S_{2}\right), \quad\left\|S_{2}\right\| \geq \beta, \\ \frac{S_{2}}{\beta}, \quad\left\|S_{2}\right\|<\beta,\end{array}\right.$

with $\operatorname{sgn}(\cdot)$ being the sign function. We set $\xi_{1}=\xi_{2}=5, \zeta_{2}=2, k_{1}=3, k_{2}=2, \lambda_{1}=1$, $\lambda_{2}=3.3, \eta_{1}=3, \eta_{2}=0.1$ and $\beta=2$ after referring to [11]. The outer-loop altitude-hold controller parameters are set as that of the proposed method. Fig. 8 illustrates that the altitude and speed are well maintained within 10 seconds for both of these two controllers, in the presence of uncertainties, and the results meet the task performance evaluation criteria. However, the curves of the throttle opening and speed imply that the SMC method might lead to severe chattering. This is unfavorable for real applications. While using the proposed controller, the undesired chattering is reduced significantly. This is because the projection-based adaptation technique is employed to estimate the uncertainties, and the uncertainties can be compensated by the estimated value in the control input. To summarize, the proposed method not only guarantees tracking performance but also yields a moderate control behavior.

\section{Conclusions}

This paper proposes a flight control approach that integrates backstepping sliding mode control with parameter adaptation for the heavyweight cargo airdrop. The controller is capable of stabilizing the plane dynamics in cargo extraction, while being robust to uncertainties of both constant and time-varying types, as well as matched and unmatched types. The method uses projection-based adaptation strategies to achieve robustness against uncertainties, and this overcomes the conservation of the SMC method that relies on the knowledge of the bounds on the complex uncertainties. The convergence and stability properties of the controller are proved by the Lyapunov theory. The control performance is evaluated on a $24,955 \mathrm{~kg}$ transport plane airdrop with an $8,000 \mathrm{~kg}$ payload. The responses of the plane satisfy the airdrop task performance criteria in the presence of pitch rate disturbance and aerodynamic uncertainty. The application of this research can be used to achieve higher levels of performance and safety in practical airdrop tasks.

The control gains are tuned in a trial and error way, which is a tedious work. The investigation of developing a criterion to specify the control parameters is interesting. This will be one of our future studies. Also, the well-known problems such as input constraints and dead zones have not been discussed in the current work. While a flight control system is actually implemented using this approach, these problems will of course have to be considered.

\section{Acknowledgements}

This work is co-supported by the National Natural Science Foundation of China (No. 61273141) and Aviation Science Foundation of China (No. 20141396012).

\section{References}

[1] Desabrais K. J., Riley J., Sadeck J., Lee C. Low-cost high-altitude low-opening cargo airdrop systems. Journal of Aircraft, Vol. 49, Issue 1, 2012, p. 349-354.

[2] Pang S. K., Ng E. Y. K., Chiu W. S. Comparison of turbulence models in near wake of transport plane C-130H fuselage. Journal of Aircraft, Vol. 50, Issue 3, 2013, p. 847-852.

[3] Gurfil P., Feldman S., Feldman M. Coordination and communication of cooperative parafoils for humanitarian aid. IEEE Transactions on Aerospace and Electronic Systems, Vol. 46, Issue 4, 2010 , p. $1747-1761$.

[4] Liu R., Sun X. X., Wang D. Heavyweight airdrop flight control design using feedback linearization and adaptive sliding mode. Transactions of the Institute of Measurement and Control, Vol. 38, Issue 10, 2016, p. 1155-1164. 
[5] Zhang J. X., Xu H. J., Zhang D. C., Liu D. L. Safety modeling and simulation of multi-factor coupling heavy-equipment airdrop. Chinese Journal of Aeronautics, Vol. 27, Issue 5, 2014, p. $1062-1069$.

[6] Liu R., Sun X. X., Dong W. H., Wang D., Chang Y. G. Dynamics modeling and L1 adaptive control of a transport aircraft for heavyweight airdrop. Mathematical Problems in Engineering, Vol. 1, 2015, p. 2015-15.

[7] Qiu Z., Xiao J., Wang S. Active-disturbance rejection control based on a novel sliding mode observer for pmsm speed and rotor position. Journal of Vibroengineering, Vol. 17, Issue 8, 2015, p. 4603-4617.

[8] Yang X. K., Zhong Y. W., Yang L. Y., Zhang J., Shen G. Z. Modeling and attitude control of aircraft with variations in mass and center of gravity. Proceedings of the 8th IEEE World Congress on Intelligent Control and Automation, IEEE, Piscataway, NJ, 2010, p. 323-329.

[9] Feng Y. L., Shi Z. K., Tang W. Dynamics modeling and control of large transport aircraft in heavy cargo extraction. Journal of Control Theory and Applications 9(2), 231-236. (2011)

[10] Kotta Ü., Tōnso M., Shumsky A. Y., Zhirabok A. N. Feedback linearization and lattice theory. Systems and Control Letters, Vol. 62, 2013, p. 248-225.

[11] Liu R., Sun X. X., Dong W. H. Dynamics modeling and control of a transport aircraft for ultra-low altitude airdrop. Chinese Journal of Aeronautics, Vol. 28, Issue 2, 2015, p. 478-487.

[12] Zhang H. Y., Shi Z. K. Variable structure control of catastrophic course in airdropping heavy cargo. Chinese Journal of Aeronautics, Vol. 22, Issue 2, 2009, p. 520-527.

[13] Plestan F., Shtessel Y., Brégeault V., Poznyak A. Sliding mode control with gain adaptation application to an electropneumatic actuator. Control Engineering Practice, Vol. 21, Issue 5, 2013, p. $679-688$.

[14] Levant A. Chattering analysis. IEEE Transaction on Automatic Control, Vol. 55, Issue 6, 2010, p. 1380-1389.

[15] Yang Y., Lu Y. P. Backstepping sliding mode control for super-low altitude heavy cargo airdrop from transport plane. Acta Aeronautica et Astronautica Sinica, Vol. 33, Issue 12, 2012, p. 2301-2312.

[16] Gu Bin, Sheng Victor S., Tay Keng Yeow, Romano Walter, Li Shuo Incremental support vector learning for ordinal regression. IEEE Transactions on Neural Networks and Learning Systems, Vol. 26, Issue 7, 2015, p. 1403-1416.

[17] Pan Zhaoqing, Zhang Yun, Kwong Sam Efficient motion and disparity estimation optimization for low complexity multiview video coding. IEEE Transactions on Broadcasting, Vol. 61, Issue 2, 2015, p. $166-176$.

[18] Wen Xuezhi, Shao Ling, Xue Yu, Fang Wei A rapid learning algorithm for vehicle classification. Information Sciences, Vol. 295, Issue 1, 2015, p. 395-406.

[19] Deng Wu, Zhao Huimin, Liu Jingjing, Yan Xiaolin, Li Yuanyuan, Yin Lifeng, Ding Chuanhua An improved CACO algorithm based on adaptive method and multi-variant strategies. Soft Computing, Vol. 19, Issue 3, 2015, p. 701-713.

[20] Xia Zhihua, Wang Xinhui, Sun Xingming, Liu Quansheng, Xiong Naixue Steganalysis of LSB matching using differences between nonadjacent pixels. Multimedia Tools and Applications, Vol. 75, Issue 4, 2016, p. 1947-1962.

[21] Sonneveldt L., Chu Q. P., Mulder J. A. Nonlinear flight control design using constrained adaptive backstepping. Journal of Guidance, Control, and Dynamics, Vol. 29, Issue 2, 2007, p. 322-336.

[22] Zou A. M., Kumar K. D., Hou Z. G. Quaternion-based adaptive output feedback attitude control of spacecraft using Chebyshev neural networks. IEEE Transactions on Neural Networks, Vol. 21, Issue 9 , 2010, p. 1457-1471.

[23] Farrell J., Sharma M., Polycarpou M. Backstepping-based flight control with adaptive function approximation. Journal of Guidance, Control, and Dynamics, Vol. 28, Issue 6, 2005, p. 1089-1102.

[24] Pomet J. B., Praly L. Adaptive nonlinear regulation: estimation from the Lyapunov equation. IEEE Transactions on Automatic Control, Vol. 37, Issue 6, 1992, p. 729-740.

[25] Natarajan V., Bentsman J. Adaptive projection-based observers and adaptive controllers for infinite dimensional systems with full-state measurement. IEEE Transactions on Automatic Control, Vol. 59, Issue 3, 2014, p. 585-598.

\section{Appendix}

The projection operator introduced in [24] bounds the estimated parameters by definition. We recall the main definitions from [24]: 
Definition A1. Consider a convex compact set with a smooth boundary given by:

$\Omega_{c}=\left\{\theta \in R^{n} \mid f(\theta) \leq c\right\}, \quad 0 \leq c \leq 1$,

where $f: R^{n} \rightarrow R$ is the following smooth convex function:

$f(\theta)=\frac{\theta^{T} \theta-\theta_{\max }^{2}}{\varepsilon_{\theta}}$,

where $\theta_{\max }$ is the norm bound imposed on the vector $\theta$, and $0<\varepsilon_{\theta}<1$ stands for the projection tolerance bound of our choice. For any given $y \in R^{n}$, the projection operator is defined as:

$\operatorname{Proj}(\theta, \mathrm{y})=\left\{\begin{array}{l}y, \quad f(\theta)<0, \\ y, \quad f(\theta) \geq 0, \quad \nabla f^{T} y \leq 0, \\ y-g(f, y), \quad f(\theta) \geq 0, \quad \nabla f^{T} y>0,\end{array}\right.$

where $\nabla f(\theta)$ is the gradient vector of $f(\cdot)$ evaluated at $\theta$, and:

$g(f, y)=\frac{\nabla f \nabla f^{T} y}{\|\nabla f\|^{2}} f(\theta)$

The properties of the projection operator are given by the following lemma:

Lemma A1. Let:

$\theta^{*} \in \Omega_{0}=\left\{\theta \in R^{n} \mid f(\theta) \leq 0\right\}$,

and let the parameter $\theta(t)$ evolve according to the following dynamics:

$\dot{\theta}(t)=\operatorname{Proj}(\theta(t), y), \quad \theta\left(t_{0}\right) \in \Omega_{c}$.

Then:

$\theta(t) \in \Omega_{c}$,

for all $t \geq t_{0}$, and:

$\left(\theta-\theta^{*}\right)^{T}(\operatorname{Proj}(\theta, y)-y) \leq 0$

Property A1. The projection operator $\operatorname{Proj}(\theta, y)$ does not alter $y$ if $\theta$ belongs to the set $\Omega_{0}$. In the set $\{0 \leq f(\theta) \leq 1\}$, $\operatorname{Proj}(\theta, y)$ subtracts a vector normal to the boundary of $\Omega_{c}$ to obtain a smooth transformation from the original vector field $y$ to an inward or tangent vector field for $c=1$. Therefore, on the boundary of $\Omega_{c}, \dot{\theta}(t)$ always points toward the inside of $\Omega_{c}$ and $\theta(t)$ never leaves the set $\Omega_{c}$.

Property A2. From the convexity of function $f(\theta)$, it follows that for any $\theta^{*} \in \Omega_{0}$ and $\theta \in \Omega_{c}$, the inequality:

$\left(\theta-\theta^{*}\right)^{T} \nabla f(\theta) \leq 0$

holds. It then follows from Definition A1 that: 
$\left(\theta-\theta^{*}\right)^{T}(\operatorname{Proj}(\theta, y)-y)=\left\{\begin{array}{l}0, \quad f(\theta)<0, \\ 0, \quad f(\theta) \geq 0, \quad \nabla f^{T} y \leq 0, \\ \underbrace{\left(\theta-\theta^{*}\right)^{T} \nabla f(\theta)}_{\leq 0} \underbrace{\nabla f^{T} y}_{\geq 0} \underbrace{f(\theta)}_{\geq 0}\end{array}, \quad f(\theta) \geq 0, \quad \nabla f^{T} y>0\right.$.

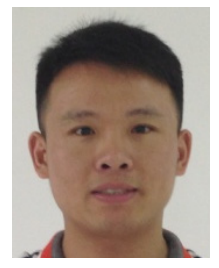

Ri Liu received M.S. degree in Aeronautics and Astronautics Engineering College from Air Force Engineering University, Xi'an, China, in 2013. Now he is pursuing the Ph.D. degree at Air Force Engineering University. His current research interests include flight dynamics and flight control.

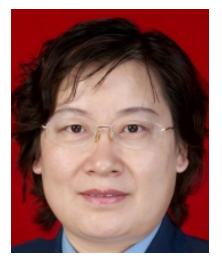

Xiuxia Sun received Ph.D. degree in Automation Institute from Beihang University, Beijing, China, in 1999. Now she works at Air Force Engineering University. Her current research interests include robust control, flight control, and flying qualities.

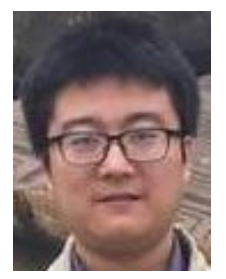

Wenhan Dong received Ph.D. degree in Aeronautics and Astronautics Engineering College from Air Force Engineering University, Xi'an, China, in 2005. Now he works at Air Force Engineering University. His current research interests include flight simulation and adaptive control.

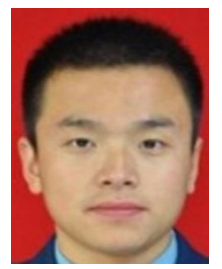

Xuning Guan received M.S. degree in Aeronautics and Astronautics Engineering College from Air Force Engineering University, Xi'an, China, in 2013. Now he works at The First Aeronautical College of Air Force. His current research interests include flight simulation and flying qualities. 